\title{
Impact of Implementing Teleophthalmology Referral Guidelines Using the eyeSmart EMR App in 63,703 Patients from India
}

\author{
Anthony Vipin Das, ${ }^{1,2}$ Rohit C. Khanna, ${ }^{3}$ Niranjan Kumar, ${ }^{3}$ and Padmaja Kumari Rani $\mathbb{D}^{2,4}$ \\ ${ }^{1}$ Department of eyeSmart EMR \& AEye, L V Prasad Eye Institute, Hyderabad, Telangana, India \\ ${ }^{2}$ Department of Teleophthalmology, L V Prasad Eye Institute, Hyderabad, Telangana, India \\ ${ }^{3}$ Gullapalli Pratibha Rao International Centre for Advancement of Rural Eye Care, L V Prasad Eye Institute, Hyderabad, \\ Telangana, India \\ ${ }^{4}$ Smt Kanuri Santhamma Centre for Vitreo Retinal Diseases, L V Prasad Eye Institute, Hyderabad, Telangana, India \\ Correspondence should be addressed to Padmaja Kumari Rani; rpk@lvpei.org
}

Received 1 October 2021; Revised 6 January 2022; Accepted 19 January 2022; Published 27 January 2022

Academic Editor: Joel Colloc

Copyright (c) 2022 Anthony Vipin Das et al. This is an open access article distributed under the Creative Commons Attribution License, which permits unrestricted use, distribution, and reproduction in any medium, provided the original work is properly cited.

\begin{abstract}
Objective. To describe the clinical indications and the impact of implementation of specific teleophthalmology referral guidelines in a large rural village vision centre network in India. Methods. This cross-sectional vision centre-based study included 1,016,284 patients presenting between January 2017 and March 2020. Patients who were referred for a teleophthalmology opinion were included as cases. The data were collected using the eyeSmart EMR app on a smart tablet. A training intervention was done to reinforce the implementation of targeted teleophthalmology referral guidelines. Results. Overall, 63,703 (6.3\%) patients were referred for a teleophthalmology opinion and were included for analysis. The median age was 41 (IQR: 26-59) years, and adults $(88.4 \%)$ were commonly referred for a consult. The two most common age groups were between $31-40$ years (17.4\%) and $21-30$ years $(16.3 \%)$, and the majority of patients were male $(59.1 \%)$. The most common clinical indication was cornea and anterior segment disorders (71.05\%). The most common queries for teleophthalmology referral before versus after the reinforcement of implementation of guidelines were red eye $(33.4 \%$ vs. $45.6 \%)$ followed by cataract $(21.2 \%$ vs. $8.1 \%)$. There was an increase in the red eye $(<0.001)$ and a decrease in cataract $(<0.001)$ which was statistically significant. The proportion of patients for whom a teleophthalmology consult could have been requested but not sent was minimal (2.3\%). Conclusion. Implementation of targeted teleophthalmology referral guidelines enables an effective triage to seek opinion for more pertinent ocular diseases that require care. Adult male patients with cornea and anterior segment disorders are most commonly referred for a teleophthalmology opinion.
\end{abstract}

\section{Introduction}

Teleophthalmology is an important tool to provide comprehensive primary eye care bridging the gaps of distance, expertise at remote rural locations. Several studies have reported the accuracy, cost-effectiveness, and patient satisfaction of the teleophthalmology model [1-3]. Barriers to the accessibility of eye care in terms of geography, time, travel, and money have been effectively addressed through effective telemedical systems worldwide $[4,5]$. Teleophthalmology models were much more cost-effective than inperson consultations in conditions such as diabetic retinop- athy and glaucoma [6, 7]. Telemedicine collaborations between developed and developing countries have shown significant benefits in the provision of expert care with positive results in conditions such as retinoblastoma $[8,9]$.

Referral guidelines of teleconsultation are crucial for a primary eye care provider to provide the appropriate management and necessary referrals to secondary/tertiary levels of care. Adherence to teleconsultation referral protocols is necessary to provide effective primary eye care. However, the lack of adherence to teleconsultation referral guidelines can result in unnecessary referrals to teleconsultation. It can also result in the missing out of referable pathology by 
the vision technician. This can be inconvenient for the patient who might incur additional, unnecessary expenses.

Our earlier pilot study of a small sample (5,604 patients) over a limited time period (1 week) showed that around $9 \%$ missed referrals for teleconsultation [10].

There was a need to see actual missed referrals in the entire cohort over longer duration and the impact of implementing targeted teleophthalmology referral guidelines.

The present study is designed to understand the adherence patterns to teleconsultation referral guidelines in a primary eye care network through an audit, before and after the implementation of targeted teleophthalmology referral guidelines. The findings of the study will aid in providing holistic primary eye care and the effective implementation of the teleophthalmology model.

\section{Materials and Methods}

2.1. Study Design, Period, Location, and Approval. This cross-sectional observational hospital-based study included all patients presenting between January 2017 and March 2020 to the village vision centres of an ophthalmology network located in 176 different geographical locations spread across 4 states (Telangana, Andhra Pradesh, Odisha, and Karnataka) of India [11]. The prevalence of disabled persons in seeing according to the 2011 census of India in erstwhile combined Andhra Pradesh (Andhra Pradesh+Telangana) was $0.47 \%(398,144 / 84,580,777)$, Odisha was $0.63 \%$ $(263,799 / 41,974,218)$, and Karnataka was $0.43 \%(264,170 /$ $61,095,297)$ [12]. The patient or the parents or guardians of the patient filled out a standard consent form for electronic data privacy at the time of registration. None of the identifiable parameters of the patient information was used for analysis of the data. The study adhered to the Declaration of Helsinki and was approved by the Institutional Ethics Committee (Hyderabad Eye Research Foundation, Hyderabad, Telangana, India). The clinical data of each patient who underwent a comprehensive ophthalmic examination was entered into an app-based electronic medical record system (eyeSmart EMR) [13] by a uniformly trained ophthalmic vision technician using a standardized template that synchronizes to the main EMR system through the cloud [14]. The vision technicians (VT) undergo a year of training at the centre of excellence and are then posted in the respective vision centres across the network. Continuous medical education modules and assessments of the vision technicians are conducted periodically through the app as well. Patients were also referred for a teleophthalmology consult through the eyeSmart app [15] and the anterior segment pictures of the eye taken by a slit lamp attachment [16]. The video conferencing tool, Skype, is used for all the teleophthalmology consults (Skype, Microsoft Corp, Redmond, USA). For optimum patient management and communication with the higher centres, a referral system is put into place whereby the VT decides on either direct referrals or teleconsultations using the eyeSmart app. The teleophthalmology advice is given by an ophthalmologist who is present at the command centre located at the tertiary eye hospital. An ophthalmologist provides advice through electronic medical record after assessing the patient complaints through electronic record data and real-time examination using video call (Skype).

2.2. Cases. A total of $1,016,284$ patients of all ages presented to the 176 vision centres of the network during the study period. Standard teleophthalmology referral guidelines were formulated which included (a) any lid abnormality such as ptosis, severe meibomitis, blepharitis, stye, chalazion, and other conditions that are obvious on torch light examination, (b) pterygium crossing the limbus, (c) pigmentation of conjunctiva or any abnormality that is clearly seen on a slit lamp, (d) red eye with or without discharge, (e) corneal scars, (f) corneal infiltrates, (g) corneal epithelial defects (need images with and without staining), (h) severe spheroidal degeneration that is close to pupillary margin or covering the pupillary area, (i) shallow anterior chamber (showing the slit view), (j) if the patient has hyperopia more than $3.0 \mathrm{Ds}$ (slit view to be shown), (k) flare and cells, (l) pupil/iris abnormalities such as atrophic patches on the iris, sphincter atrophy, new blood vessels on the iris, (m) ocular trauma, (n) any lens-related pathology which needed teleconsultation, and (o) suspected posterior segment pathology. The patients could also be referred to a higher centre for medical or surgical care.

Primary eye care providers (vision technicians) were instructed to take images as per the standard protocol of teleconsultation of the institute of all patients visiting vision centres. Currently, LVPEI anterior segment image teleconsultation protocol includes an external photo, one anterior segment image of each eye. All vision technicians have been trained in teleconsultation referral guidelines through lectures, PowerPoint slides, and WhatsApp communication periodically. These specific referral guidelines were reinforced through training programs in November 2019, and the reasons for teleophthalmology referral were compared before and after the training intervention.

The patients were classified into the following three categories: (i) patients in whom a teleophthalmology consult was sent (Category 1); (ii) patients who were referred to the secondary centre for higher care through teleophthalmology (Category 2); (iii) patients in whom the teleophthalmology consult could have been sent but was missed. These were identified based on the teleophthalmology referral guidelines (Category 3). All these categories have been compared before and after training intervention.

2.3. Data Retrieval and Processing. The data of $1,016,284$ patients included in this study were retrieved from the electronic medical record database from the eyeSmart app and segregated in a single excel sheet. The columns included the data on demographics, clinical presentation, ocular diagnosis, and reasons for teleophthalmology referral which were exported for analysis. The excel sheet with the required data was then used for analysis using the appropriate statistical software.

2.4. Statistical Analysis. Descriptive statistics using mean \pm standard deviation and median with interquartile range (IQR) were used to elucidate the demographic data. The chi- 


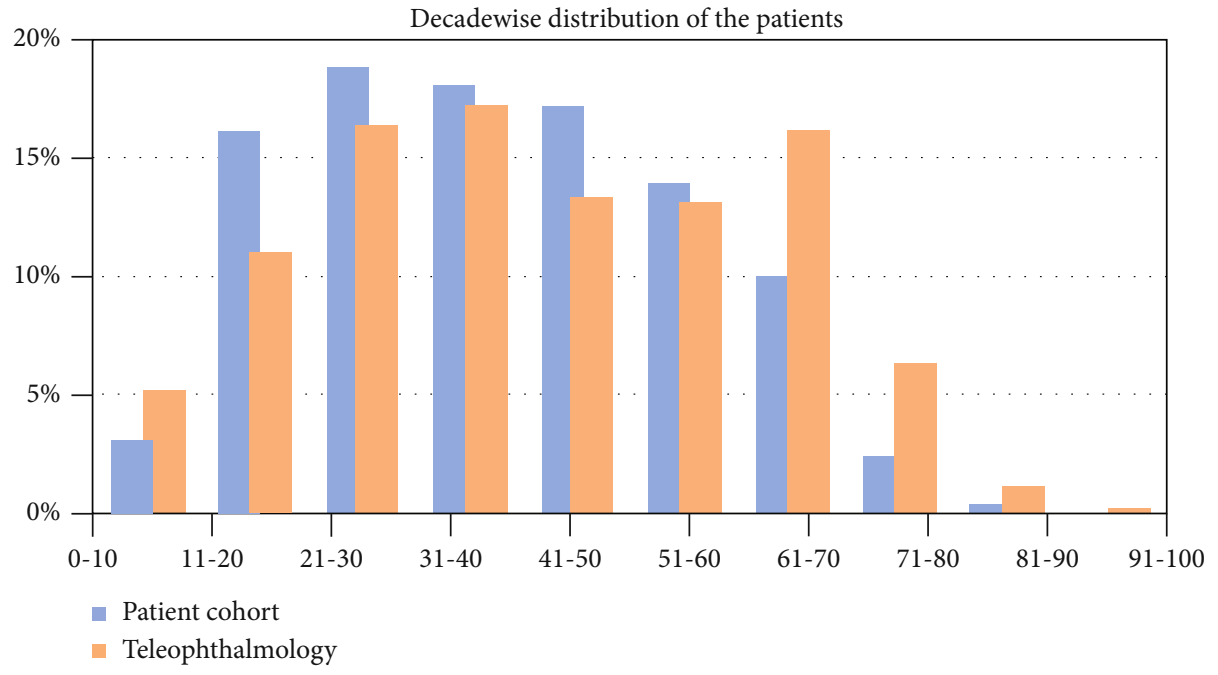

FIGURE 1: Decade-wise distribution of the overall cohort, teleophthalmology consults.

TABle 1: Patients who were referred to the secondary centre for higher care through teleophthalmology (Category 2).

\begin{tabular}{lcccc}
\hline \multirow{2}{*}{ Specialty } & \multicolumn{2}{c}{ Before } & \multicolumn{2}{c}{ After } \\
& $25723 /$ & $84.98 \%$ & $4545 /$ & 13,703 \\
& 50,000 & & $15.02 \%$ \\
\hline Cornea \& anterior & 13219 & $51.39 \%$ & 3053 & $67.17 \%$ \\
segment & 9911 & $38.53 \%$ & 923 & $20.31 \%$ \\
Cataract & 924 & $3.59 \%$ & 171 & $3.76 \%$ \\
Trauma & 766 & $2.98 \%$ & 159 & $3.50 \%$ \\
Refractive error & 399 & $1.55 \%$ & 153 & $3.37 \%$ \\
Oculoplasty & 286 & $1.11 \%$ & 39 & $0.86 \%$ \\
Retina & 102 & $0.40 \%$ & 27 & $0.59 \%$ \\
Glaucoma & 46 & $0.18 \%$ & 10 & $0.22 \%$ \\
Neuroophthalmology & 38 & $0.15 \%$ & 8 & $0.18 \%$ \\
Uvea & 32 & $0.12 \%$ & 2 & $0.04 \%$ \\
Strabismus & & & &
\end{tabular}

square test (StataCorp. 2015. Stata Statistical Software: Release 14. College Station, TX: StataCorp LP) was used for univariate analysis to detect significant differences in the reasons for referring patients for a teleophthalmology consult.

\section{Results}

3.1. Overall Cohort. A total of 1,016,284 patients presented to the village vision centre network during the study period. The mean age of the patients was $38.9 \pm 18.0$ years while the median age was 38 (IQR: 24-53) years. There were 124,327 $(12.2 \%)$ children ( $\leq 16$ years) and 891,957 (87.8\%) adults. There were 560,331 (55.1\%) male patients and 455,953 (44.9\%) female patients. The detailed decade-wise prevalence of patients is summarized in Figure 1. The most common ocular diagnosis were related to cornea and anterior segment in 486,739 (47.9\%) patients, refractive error in $346,850(34.1 \%)$ patients, and cataract in $171,244(16.9 \%)$ patients.
3.2. Teleophthalmology Cohort (Category 1). A teleophthalmology consult was sought in 63,703 (6.3\%) patients from the village vision centre network during the study period. The mean age of the patients was $41.5 \pm 19.6$ years while the median age was 41 (IQR: 26-59) years. There were $7,406(11.6 \%)$ children ( $\leq 16$ years) and $56,297(88.4 \%)$ adults. There were $37,668(59.1 \%)$ male patients and 26,035 (40.9\%) female patients. The detailed decade-wise prevalence of patients who were sent for teleophthalmology consultation is summarized in Figure 1. The most common ocular diagnosis were related to cornea and anterior segment in 45,261 (71.1\%) patients, cataract in $11,700(18.4 \%)$ patients, and oculoplasty related in 2,238 (3.5\%) patients.

Overall cohort versus teleophthalmology cohort: Figure 1 shows comparison of decade-wise prevalence percentages between the overall cohort and teleophthalmology cohort. At 21-40 years (34\%) and with increasing age more than 50 years, teleophthalmology referral is significantly more $36.8 \%$ (Tele) vs. $26.7 \%$ ((overall) $p=<0.00001$ ).

3.3. Patients Who Were Referred to the Secondary Centre for Higher Care through Teleophthalmology (Category 2). In Table 1, a total of 30,268/63,703 (47.5\%) were referred to a higher centre for medical or surgical care from the village vision centre network through teleophthalmology during the study period. The mean age of the patients was $49.2 \pm$ 19.7 years while the median age was 54 (IQR 35-66) years. There were $2,463(8.1 \%)$ children ( $\leq 16$ years) and 27,805 (91.9\%) adults. There were $17,007(56.2 \%)$ male patients and $13,261(43.8 \%)$ female patients. About 5,762 (19\%) patients who were referred from the vision centre through teleophthalmology reported to the higher centre for care. The detailed ocular diagnosis referral distribution before and after implementation is shown in Table 2.

3.4. Patients in Whom the Teleophthalmology Consult Could Have Been Sent but Was Missed (Category 3). Overall, there was a small subset of 23,809 (2.3\%) patients who could have been referred for a teleophthalmology consult and was not 
TABle 2: Ocular diagnosis distribution in patients whose teleophthalmology consultation was missed and not sent (Category 2).

\begin{tabular}{|c|c|c|c|c|}
\hline \multirow[b]{2}{*}{ Diagnosis } & \multicolumn{2}{|c|}{ Before } & \multicolumn{2}{|c|}{ After } \\
\hline & $\begin{array}{c}21429 / \\
23803\end{array}$ & $89.88 \%$ & $\begin{array}{l}2414 / \\
23803\end{array}$ & $10.12 \%$ \\
\hline Ocular surface & 11830 & $49.62 \%$ & 1406 & $5.90 \%$ \\
\hline Pterygium & 8733 & $36.63 \%$ & 1058 & $4.44 \%$ \\
\hline Allergic eye disease & 1519 & $6.37 \%$ & 203 & $0.85 \%$ \\
\hline Pinguecula & 868 & $3.64 \%$ & 87 & $0.36 \%$ \\
\hline $\begin{array}{l}\text { Subconjunctival } \\
\text { haemorrhage }\end{array}$ & 551 & $2.31 \%$ & 53 & $0.22 \%$ \\
\hline Conjunctival abrasion & 118 & $0.49 \%$ & 3 & $0.01 \%$ \\
\hline Episcleritis & 16 & $0.07 \%$ & 2 & $0.01 \%$ \\
\hline $\begin{array}{l}\text { Conjunctival } \\
\text { pigmentation }\end{array}$ & 13 & $0.05 \%$ & na & na \\
\hline Bitot spots & 12 & $0.05 \%$ & na & na \\
\hline Eyelid & 3042 & $12.76 \%$ & 310 & $1.30 \%$ \\
\hline Meibomitis & 1750 & $7.34 \%$ & 173 & $0.73 \%$ \\
\hline Stye & 927 & $3.89 \%$ & 96 & $0.40 \%$ \\
\hline Chalazion & 250 & $1.05 \%$ & 30 & $0.13 \%$ \\
\hline Ptosis & 60 & $0.25 \%$ & 9 & $0.04 \%$ \\
\hline Blepharitis & 55 & $0.23 \%$ & 2 & $0.01 \%$ \\
\hline Cornea & 2413 & $10.12 \%$ & 224 & $0.94 \%$ \\
\hline Corneal scar & 938 & $3.93 \%$ & 98 & $0.41 \%$ \\
\hline Corneal foreign body & 873 & $3.66 \%$ & 43 & $0.18 \%$ \\
\hline Corneal abrasion & 190 & $0.80 \%$ & 29 & $0.12 \%$ \\
\hline Keratitis & 123 & $0.52 \%$ & 25 & $0.10 \%$ \\
\hline Epithelial defect & 119 & $0.50 \%$ & 15 & $0.06 \%$ \\
\hline $\begin{array}{r}\text { Spheroidal } \\
\text { degeneration }\end{array}$ & 112 & $0.47 \%$ & 12 & $0.05 \%$ \\
\hline Corneal edema & 43 & $0.18 \%$ & 2 & $0.01 \%$ \\
\hline $\begin{array}{l}\text { Superficial punctate } \\
\text { keratitis }\end{array}$ & 15 & $0.06 \%$ & na & na \\
\hline Anterior segment & 1532 & $6.43 \%$ & 165 & $0.69 \%$ \\
\hline Red eye & 1532 & $6.43 \%$ & 165 & $0.69 \%$ \\
\hline Neuroophthalmology & 1188 & $4.98 \%$ & 144 & $0.60 \%$ \\
\hline Pupillary abnormality & 1141 & $4.79 \%$ & 135 & $0.57 \%$ \\
\hline Optic nerve pathology & 47 & $0.20 \%$ & 9 & $0.04 \%$ \\
\hline Retina & 442 & $1.85 \%$ & 33 & $0.14 \%$ \\
\hline $\begin{array}{l}\text { Suspect retinal } \\
\text { problem }\end{array}$ & 386 & $1.62 \%$ & 21 & $0.09 \%$ \\
\hline Coloboma & 55 & $0.23 \%$ & 11 & $0.05 \%$ \\
\hline Retinitis pigmentosa & 1 & $0.00 \%$ & 1 & $0.00 \%$ \\
\hline Strabismus & 434 & $1.82 \%$ & 70 & $0.29 \%$ \\
\hline Strabismus & 434 & $1.82 \%$ & 70 & $0.29 \%$ \\
\hline Glaucoma & 351 & $1.47 \%$ & 35 & $0.15 \%$ \\
\hline Glaucoma suspect & 281 & $1.18 \%$ & 23 & $0.10 \%$ \\
\hline Shallow AC & 68 & $0.29 \%$ & 12 & $0.05 \%$ \\
\hline Acute angle closure & 2 & $0.01 \%$ & na & na \\
\hline Trauma & 166 & $0.70 \%$ & 21 & $0.09 \%$ \\
\hline Trauma & 166 & $0.70 \%$ & 21 & $0.09 \%$ \\
\hline
\end{tabular}

TABLE 2: Continued.

\begin{tabular}{lcccc}
\hline & \multicolumn{2}{c}{ Before } & \multicolumn{2}{c}{ After } \\
Diagnosis & $21429 /$ & $89.88 \%$ & $2414 /$ & $10.12 \%$ \\
& 23803 & & 23803 & \\
\hline Uvea & 31 & $0.13 \%$ & 31 & $0.13 \%$ \\
Uveitis & 31 & $0.13 \%$ & 31 & $0.13 \%$ \\
\hline
\end{tabular}

sent by the vision technician. The detailed causes in these patients before and after implementation of specific referral guidelines is described in Table 3. About $2.6 \%$ of patients $(n=21,429 / 831,574)$ were missed referral for teleconsultation before implementation compared to $2 \%(n=2414 / 121,007)(p$ value $<0.00001)$ after implementation.

3.5. Impact of Teleophthalmology Referral Guidelines. A total of 50,000 (78.5\%) of teleophthalmology consults were done before the implementation of the specific teleophthalmology referral guidelines, and 13,703 (21.5\%) were performed after November 2019. The most common queries for teleophthalmology referral before versus after the implementation of guidelines were red eye ( $33.4 \%$ vs. $45.6 \%)$ followed by cataract $(21.17 \%$ vs $8.13 \%)$. There was an increase in the red eye $(<0.00001)$ referral and a decrease in cataract $(<0.00001)$ referral for teleophthalmology consults which was statistically significant. The detailed comparison of the ocular diagnosis before and after the implementation of the teleophthalmology guidelines is described in Table 3.

\section{Discussion}

The present study data shows that a technology-enabled primary eye care network could provide effective eye care to a million patients in three years. At the vision centre level, the vision technician utilizes the three "R's" which are Refraction, Recognize (the sight-threatening conditions), and Refer (them to the appropriate higher levels of eye care) [11].

Our network vision technician accuracy in the detection of referable anterior segment pathology has been proven [17]. In the present study, more than $90 \%$ of people who visited vision centres could be given necessary eye care at the vision centre itself. About 6.3\% required targeted referral for advice through teleophthalmology. Amongst those referred for teleophthalmology advice, more than $50 \%$ could be managed solely by teleconsultation advice at the vision centre. These patients could be given treatment advice without the need for travel to a secondary centre. A total of $30,268 / 63,703(47.5 \%)$ were referred to a higher centre for medical or surgical care from the village vision centre network through teleophthalmology during the study period. Avoiding unnecessary travel shows the advantage of teleophthalmology in providing economic impact as well as the environmental impact on reducing carbon footprint. Telemedicine has the potential to build a carbon-free health system by reducing carbon emissions associated with travel. In a systematic survey by Purohit et al., carbon footprint 
TABLE 3: Comparative description of ocular diagnosis referral before and after the implementation of specific teleophthalmology referral guidelines.

\begin{tabular}{|c|c|c|c|c|c|}
\hline \multirow{2}{*}{ Ocular diagnosis } & \multicolumn{2}{|c|}{ Before } & \multicolumn{2}{|c|}{ After } & \multirow{2}{*}{$p$ value } \\
\hline & $N$ & $\%$ & $N$ & $\%$ & \\
\hline Allergic eye disease & 2076 & $4.15 \%$ & 800 & $5.84 \%$ & $<0.00001$ \\
\hline Bitot spots & 53 & $0.11 \%$ & 17 & $0.12 \%$ & 0.572284 \\
\hline Blepharitis & 231 & $0.46 \%$ & 63 & $0.46 \%$ & 0.972703 \\
\hline Cataract & 10586 & $21.17 \%$ & 1114 & $8.13 \%$ & $<0.00001$ \\
\hline Chalazion & 386 & $0.77 \%$ & 119 & $0.87 \%$ & 0.263399 \\
\hline Coloboma & 30 & $0.06 \%$ & 6 & $0.04 \%$ & 0.479456 \\
\hline Conjunctival abrasion & 341 & $0.68 \%$ & 23 & $0.17 \%$ & $<0.00001$ \\
\hline Corneal abrasion & 312 & $0.62 \%$ & 62 & $0.45 \%$ & 0.020548 \\
\hline Corneal epithelial defect & 2221 & $4.44 \%$ & 758 & $5.53 \%$ & $<0.00001$ \\
\hline Corneal foreign body & 1093 & $2.19 \%$ & 289 & $2.11 \%$ & 0.59175 \\
\hline Corneal scar & 1302 & $2.60 \%$ & 330 & $2.41 \%$ & 0.210135 \\
\hline Corneal ulcer & 503 & $1.01 \%$ & 136 & $0.99 \%$ & 0.889236 \\
\hline Descemet membrane folds & 16 & $0.03 \%$ & 5 & $0.04 \%$ & 0.797699 \\
\hline Episcleritis & 22 & $0.04 \%$ & 10 & $0.07 \%$ & 0.180128 \\
\hline Glaucoma suspect & 49 & $0.10 \%$ & 12 & $0.09 \%$ & 0.726844 \\
\hline Headache & 513 & $1.03 \%$ & 195 & $1.42 \%$ & 0.000104 \\
\hline Meibomitis & 307 & $0.61 \%$ & 78 & $0.57 \%$ & 0.551393 \\
\hline Ocular pain & 470 & $0.94 \%$ & 180 & $1.31 \%$ & 0.000138 \\
\hline Posterior subcapsular opacification & 262 & $0.52 \%$ & 32 & $0.23 \%$ & $<0.00001$ \\
\hline Pinguecula & 507 & $1.01 \%$ & 140 & $1.02 \%$ & 0.937374 \\
\hline Pterygium & 3569 & $7.14 \%$ & 732 & $5.34 \%$ & $<0.00001$ \\
\hline Ptosis & 43 & $0.09 \%$ & 10 & $0.07 \%$ & 0.639735 \\
\hline Pupillary abnormality & 60 & $0.12 \%$ & 13 & $0.09 \%$ & 0.441594 \\
\hline Query & 3410 & $6.82 \%$ & 968 & $7.06 \%$ & 0.350478 \\
\hline Raised IOP & 32 & $0.06 \%$ & 10 & $0.07 \%$ & 0.717026 \\
\hline Red eye & 16693 & $33.39 \%$ & 6252 & $45.63 \%$ & $<0.00001$ \\
\hline Shallow anterior chamber & 30 & $0.06 \%$ & 9 & $0.07 \%$ & 0.811915 \\
\hline Spheroidal degeneration & 121 & $0.24 \%$ & 21 & $0.15 \%$ & 0.051442 \\
\hline Superficial punctate keratitis & 236 & $0.47 \%$ & 84 & $0.61 \%$ & 0.039661 \\
\hline Strabismus & 37 & $0.07 \%$ & 4 & $0.03 \%$ & 0.06704 \\
\hline Stye & 1274 & $2.55 \%$ & 406 & $2.96 \%$ & 0.009 \\
\hline Subconjunctival haemorrhage & 1403 & $2.81 \%$ & 414 & $3.02 \%$ & 0.192758 \\
\hline Suspect retinal problem & 297 & $0.59 \%$ & 47 & $0.34 \%$ & 0.000407 \\
\hline Trauma & 1470 & $2.94 \%$ & 350 & $2.55 \%$ & 0.01946 \\
\hline Uveitis & 45 & $0.09 \%$ & 14 & $0.10 \%$ & 0.678561 \\
\hline Grand total & 50000 & $100.00 \%$ & 13703 & $100.00 \%$ & \\
\hline
\end{tabular}

savings ranged from 0.70 to $372 \mathrm{~kg}$ of $\mathrm{CO}_{2}$ per consultation [18].

Our study results (Figure 1) shows that with patients of more than 50 years of age, the teleophthalmology referral was significantly more compared to their overall cohort ( $36.7 \%$ vs. $26 \% p \leq 0.00001$ ). This finding is important as older individuals could get initial advice at the primary care location through teleophthalmology without the need to travel to farther locations. This is the advantage of the tele- ophthalmology model which can address the barrier of distance and travel effectively.

Cornea and anterior segment pathology and oculoplastyrelated pathology were the predominant conditions that were given teleophthalmology care. Teleophthalmology efficacy in the detection and management of ocular surface and adnexal diseases is well proven $[10,19,20]$. Komal et al. clearly showed the cost benefits in treating corneal conditions alone through teleophthalmology were about 1200 
INR (16 US dollars) per patient; total cost saving for the community for one-year duration was 1.14 million rupees and 15,000 US dollars [5].

A slightly more number of male patients sought eye care overall and in the teleophthalmology cohort. This gender inequality in the access to eye care needs to be addressed by a root cause analysis. Similarly, it was observed that around $20 \%$ only reached higher care centres after referrals from the teleophthalmology model. The reasons for this suboptimal reach need to be explored in future studies.

There is paucity of literature regarding teleophthalmology referral guidelines and adherence patterns. The major finding of the present study is that there was more than $98 \%$ adherence to the teleophthalmology referral guidelines by the vision technicians. Only $2.3 \%$ of one million cohort patients missed their referral for a potential teleophthalmology advice. The comparison of these missed numbers, before and after training, showed that there was a significant decrease in the missed referrals for teleconsultation after training and reinforcing targeted teleophthalmology referral guidelines $(2.6 \%$ vs. $2 \%, p<0.00001)$.

Another important objective of the present study was to find the impact of reinforcing the targeted teleophthalmology referral guidelines through training of vision technicians. The most common queries for teleophthalmology referral before the reinforcement of guidelines were red eye $(33.4 \%$ vs. $45.6 \%)$ followed by cataract $(21.2 \%$ vs. $8.1 \%)$. There was an increase in the red eye $(<0.00001)$ and a decrease in cataract $(r<0.00001)$ which was statistically significant. This is important as a routine cataract does not need teleophthalmology advice and can be directly referred to a secondary centre for appropriate care, whereas a diagnosis of red eye can be effectively managed by teleconsultation alone. The major strengths of the study are the use of digital systems at the rural eye care level enables us to track the efficiency of the delivery of eye care services. The continuous monitoring of the adherence to the teleophthalmology guidelines by retrieving the digital data captured through the eyeSmart EMR app enables positive reinforcement and decreases the burden on the need for unnecessary referrals. The study in this large cohort of patients also lends insight that all patients who present to the vision centre do not require a teleophthalmology consult thereby catering to the patients who actually need the second opinion. The limitations of the study are user variability in the referral pattern across the vision centres. This is accountable to a fair extent by the training provided to them for a year before deployment and also the reinforcement of the teleophthalmology referral guidelines. The users also have a learning curve in the use of the eyeSmart EMR app and the protocol to send the pictures to the command centre. The preexisting knowledge about the use of smart phone apps by them helped to a great extent in the quick adaptation to this new technology.

To conclude, inbuilt audits and periodic training interventions to improve the adherence to targeted teleophthalmology guidelines are essential components in the implementation of the successful teleophthalmology model for effective primary eye care.

\section{Data Availability}

Access to data is restricted.

\section{Conflicts of Interest}

The authors declare that they have no conflicts of interest.

\section{Acknowledgments}

The authors wish to acknowledge the support of our Department of eyeSmart EMR and AEye team specially Mr. Ranganath Vadapalli and Mr. Mohammad Pasha. This study is funded by Hyderabad Eye Research Foundation, Hyderabad, India.

\section{References}

[1] P. Kumari Rani, R. Raman, M. Manikandan, S. Mahajan, P. G. Paul, and T. Sharma, "Patient satisfaction with teleophthalmology versus ophthalmologist-based screening in diabetic retinopathy," Journal of Telemedicine and Telecare, vol. 12, pp. 159-160, 2006.

[2] P. G. Paul, R. Raman, P. K. Rani, H. Deshmukh, and T. Sharma, "Patient satisfaction levels during teleophthalmology consultation in rural South India," Telemedicine Journal and E-Health, vol. 12, pp. 571-578, 2006.

[3] R. R. Sudhir, K. D. Frick, R. Raman, R. K. Padmaja, V. Murali, and T. Sharma, "Mobile teleophthalmology: a cost effective screening tool for diabetic retinopathy in rural South India," E-Health International, vol. 1, pp. 2-8, 2005.

[4] R. Hazin and I. Qaddoumi, "Teleoncology: current and future applications for improving cancer care globally," The Lancet Oncology, vol. 11, pp. 204-210, 2010.

[5] S. Komal, N. Radhakrishnan, A. Vardhan S, and N. V. Prajna, "Effectiveness of a teleophthalmology vision center in treating corneal disorders and its associated economic benefits," Cornea, 2021

[6] P. K. Rani, Y. Bhattarai, S. Sheeladevi, K. ShivaVaishnavi, M. H. Ali, and J. G. Babu, "Analysis of yield of retinal imaging in a rural diabetes eye care model," Indian Journal of Ophthalmology, vol. 66, pp. 233-237, 2018.

[7] N. S. Choudhari, P. Chandran, H. L. Rao et al., "LVPEI Glaucoma Epidemiology and Molecular Genetic Study: teleophthalmology screening for angle-closure disease in an underserved region," Eye, vol. 34, pp. 1399-1405, 2020.

[8] I. Qaddoumi, I. Nawaiseh, M. Mehyar et al., "Team management, twinning, and telemedicine in retinoblastoma: a 3-tier approach implemented in the first eye salvage program in Jordan," Pediatric Blood \& Cancer, vol. 51, pp. 241-244, 2008.

[9] Y. A. Yousef, I. Al-Nawaiseh, M. Mehyar et al., "How telemedicine and centralized care changed the natural history of retinoblastoma in a developing country: analysis of 478 patients," Ophthalmology, vol. 128, pp. 130-137, 2021.

[10] N. Misra, R. C. Khanna, A. L. Mettla, S. Marmamula, V. M. Rathi, and A. V. Das, "Role of teleophthalmology to manage anterior segment conditions in vision centres of South India: 
EyeSmart study-I," Indian Journal of Ophthalmology, vol. 68, pp. 362-367, 2020.

[11] G. N. Rao, S. M. Athota, P. K. Rani, R. C. Khanna, and V. Rajshekar, "Integrated model of primary and secondary eye care for underserved rural areas: the L V Prasad Eye Institute experience," Indian Journal of Ophthalmology, vol. 60, no. 5, pp. 396-400, 2012.

[12] 2022, https://censusindia.gov.in/2011census/population_ enumeration.html.

[13] P. Kammari, M. C. Ambadker, A. Loomba et al., "A novel technology tool to capture the clinical information of patients across rural vision centers in a three-tier eye care network in India using the eyeSmart EMR app," Rural and Remote Health, vol. 19, p. 5255, 2019.

[14] A. V. Das, P. Kammari, R. Vadapalli, and S. Basu, "Big data and the eyeSmart electronic medical record system - an 8year experience from a three-tier eye care network in India," Indian Journal of Ophthalmology, vol. 68, pp. 427-432, 2020.

[15] A. V. Das, S. Mididoddi, P. Kammari et al., "App-based tele ophthalmology: a novel method of rural eye care delivery connecting tertiary eye care center and vision centers in India," International Journal of Telemedicine and Applications, vol. 2019, Article ID 8107064, 6 pages, 2019.

[16] A. Loomba, S. Vempati, N. Davara et al., "Use of a tablet attachment in teleophthalmology for real-time video transmission from rural vision centers in a three-tier eye care network in India: eyeSmart cyclops," International Journal of Telemedicine and Applications, vol. 2019, Article ID 5683085, 9 pages, 2019.

[17] V. Suram, U. K. Addepalli, S. Krishnaiah, V. Kovai, and R. C. Khanna, "Accuracy of vision technicians in screening ocular pathology at rural vision centres of southern India," Clinical \& Experimental Optometry, vol. 99, pp. 183-187, 2016.

[18] A. Purohit, J. Smith, and A. Hibble, "Does telemedicine reduce the carbon footprint of healthcare? A Systematic Review," Future Healthcare Journal, vol. 8, no. 1, pp. e85-e91, 2021.

[19] Q. F. L. R. Liang, H. Liang, H. Liu, Z. Pan, C. Baudouin, and A. Labbé, "Ocular Surface Epithelial Thickness Evaluation in Dry Eye Patients: Clinical Correlations," Journal of Ophthalmology, vol. 2016, Article ID 1628469, 8 pages, 2016.

[20] M. Verma, R. Raman, and R. E. Mohan, "Application of teleophthalmology in remote diagnosis and management of adnexal and orbital diseases," Indian Journal of Ophthalmology, vol. 57, pp. 381-384, 2009. 